\title{
sciendo
}

\section{Day-to-day Variation of the Heart Rate, Heart Rate Variability, and Energy Expenditure during FIFA 11+ and Dynamic Warm-up Exercises}

\author{
by \\ Yi-Wen Chiu1, Filipe Manuel Clemente 2,3, Pedro Bezerra 2,4, Jeffrey Cayaban \\ Pagaduan ${ }^{5}$, Yung-Sheng Chen ${ }^{6,7}$
}

\begin{abstract}
There seems to be a scarcity in literature investigating day-to-day warm-up (WU) variations. We investigated day-to-day variation of psychophysiological responses during Fédération Internationale de Football Association (FIFA) $11^{+}$and dynamic warm-up (DWU) exercises. Twenty-one male collegiate soccer players were randomly assigned to the FIFA $11^{+}(n=10)$ or DWU group $(n=11)$. Each group performed the assigned WU protocol on three different occasions with a 48-h interval in between. The inter-day variation of the heart rate (HR), time distribution of HR zones, heart rate variability $(H R V)$, estimated energy expenditure (EE), blood lactate $(B L)$ concentration, and the Borg rating of perceived exertion (RPE) were recorded. A two-way repeated measures of analysis of variance was used to compare the differences in chosen variables [group (2) $\times$ day (3)]. The coefficient of variation was used to determine the dispersion of the variables over the sessions. The interclass correlation coefficient (ICC) with a two-way random model and a single measure $\left(I_{C 2}, 1\right)$ was used to determine inter-day reliability. There were no significant differences in time spent at particular HR zones and EE in both groups. The FIFA $11^{+}$exercises showed high reliability of exercise duration, average HR (HRaverage), minimal HR (HRmin), peak HR (HRpeak), and post-exercise RPE. In the DWU group, high reliability was found only in HRaverage, HR peak, and post-exercise RPE. The FIFA $11^{+}$exercises showed similar EE, exercise HR patterns, BL, and RPE compared to the DWU. The FIFA 11+ program is an intense WU protocol with a characteristically low variation of day-to-day exercise HR and RPE responses.
\end{abstract}

Key words: performance, cardiovascular responses, soccer, warm-up exercise, repeated measurement, heart rate responses.

\section{Introduction}

Strength conditioning and clinical practitioners have extensively developed warmup (WU) protocols in an attempt to improve the kinaesthetic perception and motor performance (Bishop, 2003), alleviate muscle damage (Chen et al., 2018), and minimise the risk of injuries in sports training (Emery and Meeuwisse, 2010) as well as long-term training effect (Alipasali et al., 2019). One common WU strategy is performing dynamic WU (DWU) exercises. The DWU includes concentric and eccentric actions aimed to increase muscle and core temperature (Raccuglia et al., 2016). A typical DWU involves running

\footnotetext{
1 - Department of Physical Education, Fu Jen Catholic University, New Taipei City 24206, Taiwan.

2 - Escola Superior de Desporto e Lazer, Instituto Politécnico de Viana do Castelo, Rua Escola Industrial e Comercial de

Nun'Álvares, 4900-347 Viana do Castelo, Portugal.

3 - Instituto de Telecomunicações, Delegação da Covilhã, Lisboa 1049-001, Portugal.

4 - Research Center in Sports Performance, Recreation, Innovation and Technology (SPRINT), 4960-320 Melgaço, Portugal.

5 - College of Health and Medicine, School of Health Sciences, University of Tasmania, Launceston 7250, Australia.

6 - Department of Exercise and Health Sciences, University of Taipei, Taipei 11153, Taiwan.

7 - Exercise and Health Promotion Association, New Taipei City 24156, Taiwan.
} 
with various tempos and dynamic stretching. The DWU is believed to facilitate physiological preparedness for training and competition (Andrade et al., 2015; Chatzopoulos et al., 2014; Chen et al., 2018).

In soccer, the Fédération Internationale de Football Association (FIFA) developed the FIFA 11+ protocol (FIFA $11^{+}$), which is widely used in youth (Soligard et al., 2010), collegiate (Chen et al., 2019), amateur (Nouni-Garcia et al., 2019), and professional (Daneshjoo et al., 2013) soccer players. The FIFA $11^{+}$includes 1) running exercises with dynamic stretching; 2) strength, plyometrics, and balance exercises; and 3) running exercises at high intensity. Its structure is designed with a gradual development of exercise intensity in running, agility, jumping, postural control, and core muscle strength. A single session of FIFA $11^{+}$has been found to improve vertical jump, agility, and speed (Bizzini et al., 2013; Impellizzeri et al., 2013). Furthermore, long-term implementation of FIFA $11^{+}$optimised athletic performance (Reis et al., 2013) and reduced incidence of injuries (Soligard et al., 2010; Steffen et al., 2013). Given these benefits, the persistence of physiological responses during repeated performance of the FIFA $11^{+}$remains unclear in the literature.

Given the propensity of the WU for performance enhancement, understanding day-today WU variations may provide crucial information on how daily changes in the WU affect physiological adaptation. This information is essential because players frequently engage in a standard WU protocol in daily training sessions. However, there seems to be a scarcity in literature investigating day-to-day WU variations. Thus, this study aimed to compare the heart rate (HR), HR variability (HRV), energy expenditure (EE), blood lactate (BL) concentration, and the rating of perceived exertion (RPE) between the FIFA 11+ and DWU. It was hypothesised that the FIFA $11^{+}$ would show large day-to-day variations of exercise $\mathrm{HR}, \mathrm{HRV}, \mathrm{EE}, \mathrm{BL}$, and RPE compared to the DWU exercises due to WU intensity.

\section{Methods}

\section{Participants}

Twenty-one male collegiate soccer players who met the inclusion criteria were recruited to participate. Participants had no experience in the
FIFA $11^{+}$prior to the research. The inclusion criteria were male collegiate soccer players with a training frequency of 3-5 times a week (weekly training duration of 6-9 h), no injuries reported in the last month, and participation in all data collection sessions. The exclusion criteria were 1) a history of severe neuromuscular injury; 2) current lower extremity injury; 3) cardiovascular disorder. Participants were randomly assigned to the FIFA $11^{+}(\mathrm{n}=10)$ or the DWU group $(\mathrm{n}=11)$ using a random number programme (https://www.randomizer.org) after confirmation that there were no differences in physical characteristics between the groups (Table 1). They completed an informed consent form and undertook a familiarisation session before the experiment. The experiment was conducted during the pre-competitive period, and all measurements were completed during the nontraining day. This study was approved by the local institutional ethics committee of the University of Taipei and conducted in accordance with the Declaration of Helsinki and its later amendments.

\section{Measures}

Heart rate

The minimal HR (HRmin), peak HR (HRpeak), and average HR (HRaverage) were recorded each day of the WU exercises. Time spent in particular HR zones during the WU activities was also evaluated using commercial software (Premium version 3.2, Kubios, Kuopio, Finland). HR zones were divided as follows: inactive: $<50 \%$ of the maximal HR (HRmax); very light: $50-60 \%$ of the HR max; light: $60-70 \%$ of the HR $\max$; moderate: 70 $80 \%$ of the HR $\max$; hard: $80-90 \%$ of the HRmax; and maximum: $90-100 \%$ of the HRmax. The agepredicted HR proposed by Fox and Haskell (1970) was used $\left(\mathrm{HR}_{\max }=220-\right.$ age $)$.

Heart rate variability

HRV was recorded during the FIFA $11^{+}$and DWU exercises using the portable Polar HR monitor (Polar RS800CX, Polar Electro, Kemple, Finland). The raw data of the R-R interval during the entire WU was used to derive the parasympathetic nervous system index (PNS), sympathetic nervous system index (SNS), stress index, mean R-R intervals (meanRR), standard deviation of the R-R interval (SDNN), and root mean square differences of successive R-R intervals (RMSSD). Medium artefact correction 
and smoothing set at 500 Lambda were used for HRV analysis (Tarvainen et al., 2014). The PNS was calculated based on the meanRR, RMSSD, and SD 1. The SNS was calculated based on the mean HR, Baevsky's stress index, and SD 2 (Kubios, 2021). The stress index was the result of the square root of Baevsky's stress index (Kubios, 2021).

Blood lactate

BL concentration was evaluated from the middle fingertip of blood samples taken immediately after completing the WU protocols. A blood sample was drawn into a test strip, and a lactate test analyser was used to measure BL concentration $(\mathrm{h} / \mathrm{p} /$ Cosmos Sirius, Leipzig, Germany). BL data were recorded immediately after the end of the WU exercises.

Rating of perceived exertion

A conventional 15-point Borg scale $(6=$ no exertion; $20=$ maximal exertion) was used to evaluate the RPE immediately after completing the WU exercises (Borg, 1982).

Energy expenditure

The EE was calculated using the Kubios Premium version 3.2 software (Kubios, Kuopio, Finland). Variables considered included the basal metabolic rate (BMR), diet induced thermogenesis (DIT), activity-related EE (AEE), and total EE (TEE). The BMR was calculated using the Mifflin St. Jeor equation: $9.99 \times$ weight $(\mathrm{kg})+6.25 \times$ height $(\mathrm{cm})-4.92 \times$ age +5 (Mifflin et al., 1990). The AEE was estimated using Keytel et al.'s (2005) model: $\mathrm{AEE}=\operatorname{sex}(-55.0969+0.6309 \times \mathrm{HR}+0.1988 \times$ weight $+0.2017 \times$ age $)+(1-$ sex $) \times(-20.4022+$ $0.4472 \times \mathrm{HR}-0.1263 \times$ weight $+0.074 \times$ age $)$, where male sex was 1 and female sex was 0 .

Warm-up protocols

The FIFA $11^{+}$WU consisted of 27 exercises targeting strength, balance, speed, and reaction time. The WU programme required a $25-\mathrm{m}$ distance associated with cones, markers, and balls. Participants in the FIFA $11^{+}$group performed the level 3 protocol. The duration and repetitions of each exercise in the FIFA $11^{+}$were described recently (Chen et al., 2019).

The DWU consisted of 3 min of jogging at a self-controlled pace and three sets of lower extremity exercises: 1 ) hop in and hop out; 2 ) toe walk; 3) heel walk; 4) lunge walk; 5) knee to chest; 6) heel to butt; and 7) leg swings.

\section{Design and Procedures}

The experiment included four sessions. Physical characteristics of participants (body height, body weight, and body composition) were measured during the first session. A bioelectric body composition analyser (Inbody 230, Biospace Inc., Tokyo, Japan) was used to evaluate body composition, while an electrical stadiometer (DS103, Jenix, Seoul, Korea) was used to measure body weight and height. This was followed by familiarisation with the FIFA $11^{+}$WU or DWU. Participants in the FIFA $11^{+}$group practiced using a video clip of the FIFA $11^{+}$WU exercises. During sessions two to four, participants performed the FIFA $11^{+} \mathrm{WU}$ or DWU indoors at the University of Taipei between 08:00 am and 12:00 pm. The interval between the sessions was $48 \mathrm{~h}$. The ambient temperature and relative humidity ranged from 20 to $25^{\circ} \mathrm{C}$ and from 70 to $80 \%$, respectively. To avoid the effect of circadian rhythms, participants performed the WU protocols at similar time of the day. A commercial HR monitor (Polar RS800CX, Polar Electro, Kemple, Finland) was attached to the participants' chest prior to the WU.

\section{Statistical Analyses}

Descriptive data of measured variables are presented as mean and standard error of the measurement. Statistical analyses were conducted after examining the normal distribution of each variable with the Kolmogorov-Smirnov test $(p>$ $0.05)$ and the homogeneity with the Levene's test $(p>0.05)$. The independent sample $t$ test was used to compare physical characteristics between the groups. A two-way repeated measure analysis of variance was used to compare the differences of values [group (2) $\times$ day (3)]. When a significant interaction or main effect was identified, a posthoc analysis with Bonferroni contrast was used to identify the difference between the mean values. The effect size (ES) in pairwise comparisons was tested using the Cohen $d$, which was interpreted as trivial (0.0-0.2), small (0.2-0.6), moderate (0.61.2), large (1.2-2.0), or very large (>2.0) (Hopkins et al., 2009). The coefficient of variation (CV) was used to determine variations of the variables over the three sessions. Interclass correlation coefficients (ICCs) with a two-way random model and single measure $\left(\mathrm{ICC}_{2,1}\right)$ were used to determine relative values of reliability on days 1 , 2 , and 3. The level of ICC values was expressed as 
small (0-0.30), moderate (0.31-0.49), large $(0.50-$ $0.69)$, very large $(0.70-0.89)$, or nearly perfect $(0.90-1)$ (Hopkins et al., 2009). An alpha value of $p$ $<0.05$ was set for significant differences between the means (SPSS version 16.0, IBM Corp., Armonk, NY, USA).

\section{Results}

\section{Physical Characteristics}

Participants' age, body height, body weight, body mass index, body fat content, and training experience were not significantly different between the groups $(p>0.05$; Table 1$)$.

\section{Heart Rate Variability}

The results revealed day and group interaction in the stress index $\left[F_{2,38}=3.509, \eta^{2}=\right.$ $0.156, p=0.04]$ and RMSSD $\left[F_{2}, 38=3.663, \eta^{2}=\right.$ $0.162, p=0.035]$. There were no significant differences of day and group interaction and day main effect in the other comparisons $(p>0.05)$. The post-hoc analysis revealed no significant difference of the RMSSD and stress index over the three days in both groups $(p>0.05)$ (Table 2). The day 1 and day 3 pairwise comparison showed a moderate ES of the stress index in the FIFA $11^{+}$ $(\mathrm{ES}=-0.69, p=0.07)$ and DWU groups $(\mathrm{ES}=0.83, p$ $=0.02)$. Trivial and small ESs $[-0.05(p=0.87)$ to $0.60(p=0.06)]$ were found in other pairwise comparisons.

\section{Heart Rate Zones}

There were no significant differences of day and group interaction and day main effect in each HR zone over the three days in both groups $(p>$ 0.05). The CVs of HR zones ranged from 2.89 to
$42.02 \%$ in the FIFA $11^{+}$group and 11.58 to $100 \%$ in the DWU group (Table 3). The CV of time spent on $\mathrm{HR}_{\max }$ was relatively high in the FIFA $11^{+}$and DWU groups $(42.02 \%$ and $100 \%$, respectively). Pairwise comparisons showed trivial [ES $=0.01(p$ $=0.96)$ to $0.18(p=0.73)]$ and small $[\mathrm{ES}=0.23(p=$ $0.64)$ to $-0.52(p=0.20)]$ ESs in all HR zone variables.

Energy Expenditure

There were no significant differences of day and group interaction and day main effect in the BMR, DIT, AEE, TEE over the three days in both groups $(p>0.05)$. The CV of EE was relatively low in the BMR, DIT, AEE, and TEE (from 0.21 to $4.26 \%$ ) (Table 4). Pairwise comparisons showed trivial [ES $=0.02(p=0.58)$ to $0.17(p=0.39)$ ] and small [ES $=-0.34(p=0.25)$ to $0.51(p=0.27)$ ] ES, with an expected moderate ES found on the day 2 vs. day 3 comparison of the BMR in the DWU group (ES $=-0.81 ; p=0.27$ ).

Inter-day Reliability of the Heart Rate, Blood Lactate, and Rating of Perceived Exertion

All variables showed a relatively low $\mathrm{CV}$ in both groups (Table 5). The results of the FIFA $11^{+}$ group showed high reliability of exercise duration (ICC $=0.92$, nearly perfect), HRaverage $(\mathrm{ICC}=0.92$, nearly perfect), $H R_{\min }(\mathrm{ICC}=0.91$, nearly perfect), $H_{\text {peak }}(\mathrm{ICC}=0.80$, very large), and post-exercise RPE (ICC $=0.84$, very large). A large ICC value $(0.58$, large) was found in post-exercise BL concentration. High reliability was found only in the HRaverage (ICC $=0.91$, nearly perfect), HR peak (ICC $=0.92$, nearly perfect), and post-exercise RPE (ICC $=0.91$, nearly perfect) in the DWU group.

\section{Table 1}

Physical characteristics of participants.

\begin{tabular}{lccc}
\hline & FIFA 11+ group $(\mathrm{n}=10)$ & DWU $(\mathrm{n}=11)$ & P-value \\
\hline Age $(\mathrm{yrs})$ & $20.10(0.53)$ & $18.91(0.10)$ & 0.05 \\
Body height $(\mathrm{cm})$ & $171.30(1.16)$ & $171.79(1.51)$ & 0.80 \\
Weight $(\mathrm{kg})$ & $67.32(2.02)$ & $64.27(1.99)$ & 0.30 \\
BMI & $22.95(0.69)$ & $21.81(0.73)$ & 0.28 \\
Body fat content $(\%)$ & $10.90(0.85)$ & $9.55(0.86)$ & 0.28 \\
Training experience $(\mathrm{yrs})$ & $9.90(0.41)$ & $8.73(0.38)$ & 0.05 \\
\hline
\end{tabular}

Note: Data are presented as mean and standard error of measurement. BMI: body mass index. 
Table 2

Comparisons of heart rate varaibiltiy of FIFA $11^{+}$and dynamic warm up groups at three measurement points.

\begin{tabular}{|c|c|c|c|c|c|c|c|c|}
\hline & Day 1 & Day 2 & Day 3 & CV $(\%)$ & P-value & $\begin{array}{l}\text { ES } 1 \text { vs } 2 \\
\text { ( } p \text {-value) }\end{array}$ & $\begin{array}{l}\text { ES } 1 \text { vs } 3 \\
\text { (p-value) }\end{array}$ & $\begin{array}{l}\text { ES } 2 \text { vs } 3 \\
\text { ( } p \text {-value) }\end{array}$ \\
\hline \multicolumn{9}{|l|}{ PNS } \\
\hline FIFA $11^{+}$ & $-3.17(0.19)$ & $-3.26(0.17)$ & $-3.32(0.18)$ & 2.41 & 0.39 & 0.16 trivial $(.52)$ & 0.27 small (.08) & 0.12 trivial $(.58)$ \\
\hline DWU & $-3.62(0.16)$ & $-3.54(0.15)$ & $-3.49(0.15)$ & 1.79 & 0.58 & -0.15 trivial $(.55)$ & -0.25 small $(.34)$ & -0.11 trivial $(.64)$ \\
\hline \multicolumn{9}{|l|}{ SNS } \\
\hline FIFA $11^{+}$ & $6.37(0.59)$ & $7.05(0.94)$ & $7.22(0.69)$ & 6.54 & 0.29 & -0.27 small $(.29)$ & -0.42 small $(.07)$ & -0.07 trivial $(.79)$ \\
\hline DWU & $9.64(0.69)$ & 9.19 (1.09) & $8.19(0.95)$ & 8.21 & 0.16 & -0.15 trivial $(.62)$ & -0.53 small $(.05)$ & 0.29 small (.21) \\
\hline
\end{tabular}

Stress index

\begin{tabular}{|c|c|c|c|c|c|c|c|c|}
\hline FIFA $11^{+}$ & $19.29(2.30)$ & $23.65(3.19)$ & $24.08(2.10)$ & 11.86 & 0.15 & -0.50 small $(.16)$ & -0.69 moderate $(.07)$ & -0.05 small $(.87)$ \\
\hline DWU & 34.09 (1.59) & $32.09(3.88)$ & $27.80(2.81)$ & 10.26 & 0.17 & 0.20 small (.60) & 0.83 moderate $(.02)$ & 0.38 small $(.26)$ \\
\hline
\end{tabular}

MeanRR (ms)

\begin{tabular}{|c|c|c|c|c|c|c|c|c|}
\hline FIFA $11^{+}$ & 468.35 (9.97) & $476.60(14.74)$ & $472.34(15.19)$ & 0.87 & 0.70 & -0.21 small (.37) & -0.10 trivial $(.72)$ & 0.09 trivial $(.67)$ \\
\hline DWU & 451.05 (16.71) & 454.02 (15.77) & $463.41(17.16)$ & 1.42 & 0.56 & -0.06 trivial $(.81)$ & -0.22 small $(.36)$ & -0.17 trivial $(.42)$ \\
\hline
\end{tabular}

SDNN (ms)

\begin{tabular}{|c|c|c|c|c|c|c|c|c|}
\hline FIFA $11^{+}$ & $17.16(3.08)$ & $14.82(2.06)$ & $14.09(2.06)$ & 10.43 & 0.33 & 0.28 small $(.41)$ & 0.37 small (.15) & 0.11 trivial $(.61)$ \\
\hline DWU & $6.86(0.57)$ & $7.77(0.75)$ & $8.40(0.94)$ & 10.34 & 0.14 & -0.41 small $(.27)$ & -0.60 small $(.06)$ & -0.22 small $(.41)$ \\
\hline
\end{tabular}

RMSSD (log)

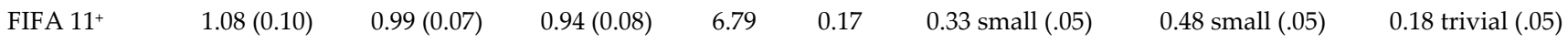

DWU $\quad 0.73(0.04) \quad 0.81(0.45) \quad 0.80(0.04) \quad 5.69 \quad 0.19 \quad-0.57$ small $(.14) \quad-0.53$ small $(.12) \quad 0.06$ trivial $(.86)$

Note: Data are presented as mean and standard error of measurement. $C V=$ coefficient of variation; ES = effect size; $D W U=$ dynamic warm up group; $P N S=$ parasympathetic nervous system index; SNS = sympathetic nervous system index; MeanRR = mean $R-R$ intervals; $S D N N=$ standard deviation of $R-R$ intervals; $R M S S D=$ root mean square differences of successive $R-R$ intervals. 
Table 3

Comparisons of time spent in particular heart rate zones during FIFA11+ and dynamic warm-up exercises at three measurement points.

\begin{tabular}{|c|c|c|c|c|c|c|c|c|}
\hline & Day 1 & Day 2 & Day 3 & CV (\%) & $\mathrm{P}$-value & $\begin{array}{l}\text { ES } 1 \text { vs } 2 \text { ( } p- \\
\text { value) }\end{array}$ & $\begin{array}{l}\text { ES } 1 \text { vs } 3 \\
\text { ( } p \text {-value) }\end{array}$ & $\begin{array}{l}\text { ES } 2 \text { vs } 3 \\
\text { ( } p \text {-value) }\end{array}$ \\
\hline \multicolumn{9}{|c|}{ Inactive (\%) } \\
\hline FIFA $11^{+}$ & $10.81(3.72)$ & $11.56(4.31)$ & $10.57(4.25)$ & 4.73 & 0.93 & $\begin{array}{c}-0.06 \text { trivial } \\
(.81)\end{array}$ & 0.02 trivial $(.91)$ & 0.07 trivial $(.74)$ \\
\hline DWU & $4.96(2.50)$ & $2.45(1.10)$ & $4.13(2.19)$ & 33.13 & 0.59 & 0.42 small (.36) & 0.11 trivial $(.78)$ & -0.30 small $(.27)$ \\
\hline \multicolumn{9}{|c|}{$\begin{array}{l}\text { Very light } \\
(\%)\end{array}$} \\
\hline FIFA $11^{+}$ & $23.78(3.59)$ & $25.12(3.73)$ & $24.86(3.51)$ & 2.89 & 0.88 & $\begin{array}{c}-0.12 \text { trivial } \\
(.61)\end{array}$ & $\begin{array}{c}-0.01 \text { trivial } \\
(.73)\end{array}$ & 0.02 trivial (.93) \\
\hline DWU & $20.56(6.88)$ & $24.54(7.73)$ & $34.29(9.38)$ & 26.79 & 0.29 & $\begin{array}{c}-0.17 \text { trivial } \\
(.13)\end{array}$ & -0.52 small $(.20)$ & -0.36 small (.49) \\
\hline \multicolumn{9}{|l|}{ Light (\%) } \\
\hline FIFA $11^{+}$ & $23.49(1.82)$ & $23.64(2.02)$ & $25.69(2.43)$ & 5.07 & 0.44 & $\begin{array}{c}-0.02 \text { trivial } \\
(.95)\end{array}$ & -0.32 small (.09) & -0.29 small (.33) \\
\hline DWU & $33.16(5.98)$ & $39.86(8.78)$ & $30.84(6.73)$ & 13.52 & 0.33 & -0.28 small $(.23)$ & 0.12 trivial $(.76)$ & 0.37 small $(.24)$ \\
\hline \multicolumn{9}{|l|}{$\begin{array}{l}\text { Moderate } \\
(\%)\end{array}$} \\
\hline FIFA $11^{+}$ & $22.24(1.69)$ & $20.77(2.88)$ & $20.58(2.05)$ & 4.27 & 0.77 & 0.20 trivial $(.54)$ & 0.28 small (.52) & 0.02 trivial (.95) \\
\hline DWU & $26.93(7.52)$ & $21.33(6.49)$ & $17.84(6.00)$ & 20.81 & 0.36 & 0.25 small $(.27)$ & 0.43 small (.14) & 0.18 trivial $(.73)$ \\
\hline \multicolumn{9}{|l|}{ Hard (\%) } \\
\hline FIFA $11^{+}$ & $17.69(2.75)$ & $15.06(2.12)$ & $16.36(3.44)$ & 8.03 & 0.72 & 0.34 small (.28) & 0.13 trivial $(.74)$ & $\begin{array}{c}-0.14 \text { trivial } \\
(.69)\end{array}$ \\
\hline DWU & $14.39(7.85)$ & $11.42(7.68)$ & $12.73(8.34)$ & 11.58 & 0.54 & 0.12 trivial $(.30)$ & 0.07 trivial $(.56)$ & $\begin{array}{c}-0.05 \text { trivial } \\
(.59)\end{array}$ \\
\hline \multicolumn{9}{|c|}{$\begin{array}{l}\text { Maximum } \\
(\%)\end{array}$} \\
\hline FIFA $11^{+}$ & $1.99(0.96)$ & $3.84(1.97)$ & $1.94(1.42)$ & 42.02 & 0.20 & -0.37 small $(.15)$ & 0.01 trivial $(.96)$ & 0.35 small (.19) \\
\hline DWU & $0(0)$ & $0.39(0.39)$ & $0.17(0.17)$ & 100 & 0.56 & - & - & 0.23 small (.64) \\
\hline
\end{tabular}

Note: Data are presented as mean and standard error of measurement. $C V=$ coefficient of variation; ES = effect size; $D W U=$ dynamic warm up group; Inactive $=<50 \%$ of the maximal heart rate; Very light $=50-60 \%$ of the maximal heart rate; Light $=60-70 \%$ of the maximal heart rate; Moderate $=70-80 \%$ of the maximal heart rate; Hard $=80-90 \%$ of the maximal heart rate; Maximum $=90-100 \%$ of the maximal heart rate. 
Table 4

Comparisons of energy expenditure during FIFA11+ and dynamic warm up exercises at three measurement points.

$\begin{array}{lllllll} & & & & & \text { ES 1 vs } 2 & \text { ES 1 vs 3 } \\ \text { Day } 1 & \text { Day } 2 & \text { Day } 3 & \begin{array}{l}\text { CV } \\ (\%)\end{array} & \text { P-value } & (p \text {-value }) & \text { ES 2 vs 3 } \\ & & & & \end{array}$

$\operatorname{BMR}(\mathrm{kcal} / \mathrm{min})$

$\begin{array}{lllllllll}\text { FIFA } 11^{+} & 23.98(0.95) & 24.48(0.95) & 24.87(0.69) & 1.81 & 0.38 & -0.02 \text { trivial }(.44) & -0.34 \text { small }(.25) & -0.15 \text { trivial }(.47) \\ & & & & & & & & \\ \text { DWU } & 21.12(0.34) & 20.61(0.30) & 21.79(0.58) & 2.81 & 0.27 & 0.51 \text { small }(.27) & -0.45 \text { small }(.56) & -0.81 \text { moderate }\end{array}$

$\operatorname{DIT}(\mathrm{kcal} / \mathrm{min})$

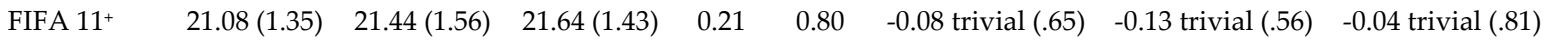

DWU $\quad 20.07(1.45) \quad 19.93(1.40) \quad 19.32(1.36) \quad 2.02 \quad 0.60 \quad 0.03$ trivial $(.55) \quad 0.17$ trivial $(.39) \quad 0.14$ trivial $(.67)$

$\operatorname{AEE}(\mathrm{kcal} / \mathrm{min})$

\begin{tabular}{|c|c|c|c|c|c|c|c|c|}
\hline FIFA $11^{+}$ & $\begin{array}{l}165.76 \\
(11.45)\end{array}$ & $\begin{array}{l}168.49 \\
(13.78)\end{array}$ & $\begin{array}{l}169.93 \\
(12.57)\end{array}$ & 1.26 & 0.85 & -0.07 trivial $(.70)$ & -0.11 trivial $(.62)$ & -0.04 trivial $(.85)$ \\
\hline DWU & $\begin{array}{l}159.55 \\
(13.08)\end{array}$ & $\begin{array}{l}158.72 \\
(12.57)\end{array}$ & $\begin{array}{l}156.15 \\
(11.38)\end{array}$ & 1.12 & 0.81 & 0.02 trivial $(.58)$ & 0.09 trivial (.64) & 0.07 trivial $(.97)$ \\
\hline
\end{tabular}

$\operatorname{TEE}(\mathrm{kcal} / \mathrm{min})$

\begin{tabular}{|c|c|c|c|c|c|c|c|c|}
\hline FIFA $11^{+}$ & $\begin{array}{l}210.83 \\
(13.51)\end{array}$ & $\begin{array}{l}214.41 \\
(15.63)\end{array}$ & $\begin{array}{l}216.44 \\
(14.27)\end{array}$ & 0.21 & 0.80 & -0.08 trivial $(.65)$ & -0.13 trivial $(.56)$ & -0.04 trivial $(.81)$ \\
\hline
\end{tabular}

Note: Data are presented as mean and standard error of measurement. $C V=$ coefficient of variation; $E S=$ effect size; $D W U=$ dynamic warm up group; $B M R=$ basal metabolic rate; $D I T=$ diet induced thermogenesis; $A E E=$ activity-related exergy expenditure; $T E E=$ total exergy expenditure. 
Table 5

Inter-day reliability of the rating of perceived exertion, blood lactate concentration, and exercise heart rate during FIFA11 ${ }^{+}$and dynamic warm up exercises at three separate measurement points.

\begin{tabular}{lcccccc}
\hline & Day 1 & Day 2 & Day 3 & CV (\%) $p$-value & ICC (95\% CI) \\
\hline Exercise duration (min) & & & & & & \\
& & & & & & \\
FIFA 11+ & $21.45(0.50)$ & $21.82(0.38)$ & $21.58(0.47)$ & 0.87 & 0.43 & $0.92(0.78-0.98)$ nearly perfect \\
DWU & $18.55(0.16)$ & $18.21(0.06)$ & $18.90(0.40)$ & 1.86 & 0.22 & $0.31(0.29-0.61)$ moderate
\end{tabular}

Exercise HRaverage (bpm)

\begin{tabular}{|c|c|c|c|c|c|c|}
\hline FIFA $11^{+}$ & $128.65(2.84)$ & $127.02(4.08)$ & $128.14(3.89)$ & 0.65 & 0.78 & $0.92(0.79-0.98)$ nearly perfect \\
\hline DWU & $134.83(4.88)$ & $133.83(4.84)$ & $131.30(4.98)$ & 1.36 & 0.56 & $0.91(0.76-0.97)$ nearly perfect \\
\hline
\end{tabular}

Exercise $\mathrm{HR}_{\min }(\mathrm{bpm})$

\begin{tabular}{|c|c|c|c|c|c|c|}
\hline FIFA $11^{+}$ & $75.90(3.76)$ & $79.21(4.58)$ & $79.54(4.18)$ & 2.57 & 0.39 & $0.91(0.74-0.98)$ nearly perfect \\
\hline DWU & $89.40(2.86)$ & $84.29(3.68)$ & $86.77(3.40)$ & 2.94 & 0.37 & $0.70(0.18-0.91)$ very large \\
\hline
\end{tabular}

Exercise $\mathrm{HR}_{\text {peak }}(\mathrm{bpm})$

\begin{tabular}{|c|c|c|c|c|c|c|}
\hline FIFA $11^{+}$ & $178.65(2.27)$ & $177.71(2.36)$ & $177.07(3.45)$ & 0.45 & 0.72 & $0.80(0.43-0.95)$ very large \\
\hline DWU & $153.96(5.14)$ & $153.42(5.38)$ & $153.62(5.55)$ & 0.18 & 0.99 & $0.92(0.80-0.98)$ nearly perfect \\
\hline
\end{tabular}

Post-exercise BL (mmol/L)

$\begin{array}{lllllll}\text { FIFA } 11^{+} & 7.81(0.77) & 7.73(0.81) & 7.73(0.76) & 0.60 & 0.99 & 0.58(-0.27-0.90) \text { large } \\ \text { DWU } & 5.39(0.86) & 5.08(0.85) & 5.51(0.87) & 4.16 & 0.85 & 0.64(-0.07-0.91) \text { large }\end{array}$

Post-exercise RPE (a.u.)

\begin{tabular}{|c|c|c|c|c|c|c|}
\hline FIFA $11^{+}$ & $14.10(0.66)$ & $14.30(0.73)$ & $13.00(0.54)$ & 5.07 & 0.65 & $0.84(0.47-0.95)$ very large \\
\hline DWU & $12.55(0.81)$ & $11.82(0.62)$ & $12.36(0.53)$ & 4.27 & 0.26 & $0.91(0.76-0.97)$ nearly perfect \\
\hline
\end{tabular}

Note: Data are presented as mean and standard error of measurement. $C V=$ coefficient of variation; $I C C=$ interclass correlation coefficients; $C I=$ confident interval; $D W U=$ dynamic warm up group; $H R_{\text {min }}=$ minimal heart rate; $H R_{\text {peak }}=$ peak heart rate; $H R_{\text {average }}=$ average heart rate; $B L=$ blood lactate concentration; $R P E=$ rating of perceived exertion; $b p m=$ beat per minute; $\mathrm{mmol} / \mathrm{L}=$ millimoles per litre. 


\section{Discussion}

This is the first study to examine inter-day variations of the exercise $H R$, exercise $H R V$, time distribution of HR zones, and EE during the FIFA $11^{+}$and DWU exercises. The major findings of the present study were consistent psychophysiological responses and energy cost during the FIFA $11^{+}$and DWU exercises over three different days. Furthermore, the FIFA $11^{+}$ WU showed high reliability in exercise duration, $H_{\text {min, }} H_{\text {average, }} \mathrm{HR}_{\text {peak, }}$ and the post-exercise RPE. However, the DWU showed high reliability measures in HRaverage, $\mathrm{HR}_{\text {peak, }}$ and the post-exercise RPE. Low CVs over the three-day measurements indicated similarity of the cardio-related responses, BL concentration, and perceived exertion in day-to-day performance of the FIFA $11^{+} \mathrm{WU}$ protocols.

Interestingly, we found no statistical differences in inter-day HR-based physiological demands, cost of EE, and exercise profiles in the FIFA $11^{+}$and DWU groups. This finding indicates that the exercise HR, exercise HRV, and AEE were repeatable in both WU schemes. However, a large $\mathrm{CV}$ of time spent in particular HR zones was found in the DWU group. In contrast, the FIFA $11^{+}$ group demonstrated a low CV of time spent in particular HR zones, which may be related to the specific structure and standardised instructions of the FIFA $11^{+}$WU.

Our study demonstrated that the FIFA $11^{+}$ WU imposed a larger proportion of high and low intensities of $\mathrm{HR}$ responses than the DWU. Additionally, the HReak during the FIFA $11^{+}$WU was about $177-178$ beats/min, indicating that the exercise intensity of the FIFA $11^{+}$WU can approach to $85-90 \%$ of the HRmax. The high intensity of the FIFA $11^{+}$WU was related to highspeed running exercises in the third part of the programme. However, the HRaverage $\left(\right.$ FIFA $11^{+}=127$ - 129 beats/min vs. DWU $=131-134$ beats/min) was lower during the FIFA $11^{+} \mathrm{WU}$ and associated with more time spent in the HR zone at inactive intensity $\left(\right.$ FIFA $11^{+}=10.57-11.56 \%$ vs. DWU $=$ $2.45-4.96 \%)$ compared to that during the DWU. This observation indicates adequate recovery time while performing the FIFA $11^{+}$WU. HR patterns observed in the FIFA $11^{+}$WU and DWU suggest the use of an appropriate WU protocol for the physical demand of technical/tactical training sessions, which means that, for example, if the technical/training intensity is high, the FIFA $11^{+}$ WU is suitable.

The FIFA $11^{+}$group demonstrated high reliability of exercise duration, HRmin, HRaverage, $\mathrm{HR}_{\text {peak, }}$ and the post-exercise RPE, but the interday reliability of post-exercise BL concentration was large. In contrast, the DWU group showed excellent ICC values of the HRaverage, HR peak, and post-exercise RPE, but the ICC value of postexercise BL concentration was large. Exercise duration was not consistent during the DWU over the three days of repeated performance due to a poor ICC value (0.31, moderate), indicating a large intra-subject variation. Absence of practical information regarding day-to-day variation and reliability of WU exercise activities in the literature makes the results incomparable among the studies. Nevertheless, very large and nearly large ICC values found in the HR patterns and RPE serve as useful and reliable evaluation of internal loads monitoring WU exercises. Since the exercise HR is a reliable variable to estimate the intensity of exercise and it is affected by training status or the competition schedule (Buchheit, 2014), the FIFA $11^{+}$WU and DWU can be used as a standard WU protocol to control the WU intensity in a daily-based WU routine. Future studies should investigate the effect of WU intensity on exercise performance in the subsequent training sessions.

The campaigning of WU regimes was based on cross-sectional studies (Chatzopoulos et al., 2014; Chen et al., 2018; Sanchez-Sanchez et al., 2018). Ayala et al. (2017) reported that dynamic WU activities resulted in the optimal hamstrings and quadriceps muscle strength ratio in comparison with the FIFA $11^{+}$programme. The possible explanation of the inferior effect of the FIFA $11^{+}$WU is that the programme does not include resistance-based activities. Thus, activity profiles of the WU protocol may be a crucial factor for benefits of WU effects (Murphy et al., 2010; Pagaduan et al., 2012). Recently, we found no difference of WU effects in the drop jump and maximal muscle strength between the FIFA $11^{+}$ WU and DWU (Chen et al., 2019). The present findings further support the notions of similar characteristics of cardiac-related responses during the FIFA $11^{+}$WU and DWU and that both WU protocols can be valid alternatives for coaches and practitioners. 
The first study limitation was that participants were not exposed to a crossover condition. This could undermine the true interpretation of individual variability. The second limitation was that HR recovery after the WU was not recorded. Since HR recovery can be compromised with training loads and fatigue status after submaximal exercise (Meur et al., 2017), the outcome measure of post-WU HR recovery can provide practical information for subsequent activities during training sessions. Lastly, running speed was not recorded during the exercises. Acquisition of running data may help explain variability in the results.

\section{Conclusions}

The FIFA $11^{+}$WU showed similar characteristics of inter-day variants of EE, exercise HR patterns, BL concentration, and RPE to the DWU. It is an intense WU protocol with characteristically low variation of day-to-day exercise HR responses, $\mathrm{AEE}$, exercise intensity, exercise duration, and psychological strain. Our study findings suggest the alternative use of the FIFA $11^{+}$WU and DWU as daily routine $\mathrm{WU}$ protocols in male collegiate soccer players.

\section{References}

Alipasali, F., Papadopoulou, S. D., Gissis, I., Komsis, G., Komsis, S., Kyranoudis, A., Knechtle, B., \& Nikolaidis, P. T. (2019). The effect of static and dynamic stretching exercises on sprint ability of recreational male volleyball players. International Journal of Environmental Research and Public Health, 16(16), 2835. https://doi.org/10.3390/ijerph16162835

Andrade, D. C., Henriquez-Olguín, C., Beltrán, A. R., Ramírez, M. A., Labarca, C., Cornejo, M., Álvarez, C., \& Ramírez-Campillo, R. (2015). Effects of general, specific and combined warm-up on explosive muscular performance. Biology of Sport, 32(2), 123-128. https://doi.org/10.5604/20831862.1140426

Ayala, F., Calderón-López, A., Delgado-Gosálbez, J. C., Parra-Sánchez, S., Pomares-Noguera, C., HernándezSánchez, S., López-Valenciano, A., \& De Ste Croix, M. (2017). Acute effects of three neuromuscular warm-up strategies on several physical performance measures in football players. PloS one, 12(1), e0169660. https://doi.org/10.1371/journal.pone.0169660

Bishop D. (2003). Warm up I: potential mechanisms and the effects of passive warm up on exercise performance. Sports Medicine, 33(6), 439-454. https://doi.org/10.2165/00007256-200333060-00005

Bizzini, M., Impellizzeri, F. M., Dvorak, J., Bortolan, L., Schena, F., Modena, R., \& Junge, A. (2013). Physiological and performance responses to the "FIFA 11+" (part 1): is it an appropriate warmup?. Journal of Sports Sciences, 31(13), 1481-1490.

Borg G. A. (1982). Psychophysical bases of perceived exertion. Medicine and Science in Sports and Exercise, 14(5), 377-381.

Buchheit M. (2014). Monitoring training status with HR measures: do all roads lead to Rome?. Frontiers in Physiology, 5, 73. https://doi.org/10.3389/fphys.2014.00073

Chatzopoulos, D., Galazoulas, C., Patikas, D., \& Kotzamanidis, C. (2014). Acute effects of static and dynamic stretching on balance, agility, reaction time and movement time. Journal of Sports Science $\mathcal{E}$ Medicine, 13(2), 403-409.

Chen, C. H., Chen, Y. S., Wang, Y. T., Tseng, W. C., \& Ye, X. (2018). Effects of preconditioning hamstring resistance exercises on repeated sprinting-induced muscle damage in female soccer players. Biology of Sport, 35(3), 269-275. https://doi.org/10.5114/biolsport.2018.77827

Chen, C. H., Ye, X., Wang, Y. T., Chen, Y. S., \& Tseng, W. C. (2018). Differential effects of different warm-up protocols on repeated sprints-induced muscle damage. Journal of Strength and Conditioning Research, 32(11), 3276-3284.

Chen, Y. S., Lai, W. L., Hou, C. W., Chen, C. H., Chiu, Y. W., \& Bezzera, P. (2019). Effect of post warm-up resting interval on static and dynamic balance, and maximal muscle strength followed by the FIFA 11+ and dynamic warm-up exercises. The Journal of Sports Medicine and Physical Fitness, 59(3), 366375. https://doi.org/10.23736/S0022-4707.18.08247-6 
Daneshjoo, A., Mokhtar, A. H., Rahnama, N., \& Yusof, A. (2013). Effects of the 11+ and Harmoknee warm-up programs on physical performance measures in professional soccer players. Journal of Sports Science E Medicine, 12(3), 489-496.

Emery, C. A., \& Meeuwisse, W. H. (2010). The effectiveness of a neuromuscular prevention strategy to reduce injuries in youth soccer: a cluster-randomised controlled trial. British Journal of Sports Medicine, 44(8), 555-562. https://doi.org/10.1136/bjsm.2010.074377

Hopkins, W. G., Marshall, S. W., Batterham, A. M., \& Hanin, J. (2009). Progressive statistics for studies in sports medicine and exercise science. Medicine and Science in Sports and Exercise, 41(1), 3-13. https://doi.org/10.1249/MSS.0b013e31818cb278

Fox, S. M., \& Haskell, W. L. (1970). The exercise stress test. Needs for standardization. In Cardiology. Current Topics and Progress. Eliakim, M., Neufeld, H.N., Eds. Academic Press: New York.

Impellizzeri, F. M., Bizzini, M., Dvorak, J., Pellegrini, B., Schena, F., \& Junge, A. (2013). Physiological and performance responses to the FIFA 11+ (part 2): a randomised controlled trial on the training effects. Journal of Sports Sciences, 31(13), 1491-1502. https://doi.org/10.1080/02640414.2013.80292

Keytel, L. R., Goedecke, J. H., Noakes, T. D., Hiiloskorpi, H., Laukkanen, R., van der Merwe, L., \& Lambert, E. V. (2005). Prediction of energy expenditure from heart rate monitoring during submaximal exercise. Journal of Sports Sciences, 23(3), 289-297. https://doi.org/10.1080/02640410470001730089

Kubios. HRV in evaluating ANS function. https://www.kubios.com/hrv-ans-function/

Le Meur, Y., Buchheit, M., Aubry, A., Coutts, A. J., \& Hausswirth, C. (2017). Assessing Overreaching With Heart-Rate Recovery: What Is the Minimal Exercise Intensity Required?. International Journal of Sports Physiology and Performance, 12(4), 569-573. https://doi.org/10.1123/ijspp.2015-0675

Mifflin, M. D., St Jeor, S. T., Hill, L. A., Scott, B. J., Daugherty, S. A., \& Koh, Y. O. (1990). A new predictive equation for resting energy expenditure in healthy individuals. The American Journal of Clinical Nutrition, 51(2), 241-247. https://doi.org/10.1093/ajcn/51.2.241

Murphy, J. R., Di Santo, M. C., Alkanani, T., \& Behm, D. G. (2010). Aerobic activity before and following short-duration static stretching improves range of motion and performance vs. a traditional warmup. Applied Physiology, Nutrition, and Metabolism, 35(5), 679-690. https://doi.org/10.1139/H10-062

Nouni-Garcia, R., Asensio-Garcia, M. R., Orozco-Beltran, D., Lopez-Pineda, A., Gil-Guillen, V. F., Quesada, J. A., Bernabeu Casas, R. C., \& Carratala-Munuera, C. (2019). The FIFA 11 programme reduces the costs associated with ankle and hamstring injuries in amateur Spanish football players: A retrospective cohort study. European Journal of Sport Science, 19(8), 1150-1156. https://doi.org/10.1080/17461391.2019.1577495

Pagaduan, J. C., Pojskić, H., Užičanin, E., \& Babajić, F. (2012). Effect of various warm-up protocols on jump performance in college football players. Journal of Human Kinetics, 35, 127-132. https://doi.org/10.2478/v10078-012-0086-5

Raccuglia, M., Lloyd, A., Filingeri, D., Faulkner, S. H., Hodder, S., \& Havenith, G. (2016). Post-warm-up muscle temperature maintenance: blood flow contribution and external heating optimisation. European Journal of Applied Physiology, 116(2), 395-404. https://doi.org/10.1007/s00421015-3294-6

Reis, I., Rebelo, A., Krustrup, P., \& Brito, J. (2013). Performance enhancement effects of Fédération Internationale de Football Association's "The 11+" injury prevention training program in youth futsal $\begin{array}{llll}\text { players. Clinical Journal of } & \text { 318-320. }\end{array}$ https://doi.org/10.1097/JSM.0b013e318285630e

Sanchez-Sanchez, J., Rodriguez, A., Petisco, C., Ramirez-Campillo, R., Martínez, C., \& Nakamura, F. Y. (2018). Effects of different post-activation potentiation warm-ups on repeated sprint ability in soccer players from different competitive levels. Journal of Human Kinetics, 61, 189-197. https://doi.org/10.1515/hukin-2017-0131 
Soligard, T., Nilstad, A., Steffen, K., Myklebust, G., Holme, I., Dvorak, J., Bahr, R., \& Andersen, T. E. (2010). Compliance with a comprehensive warm-up programme to prevent injuries in youth football. British Journal of Sports Medicine, 44(11), 787-793. https://doi.org/10.1136/bjsm.2009.070672

Steffen, K., Meeuwisse, W. H., Romiti, M., Kang, J., McKay, C., Bizzini, M., Dvorak, J., Finch, C., Myklebust, G., \& Emery, C. A. (2013). Evaluation of how different implementation strategies of an injury prevention programme (FIFA 11+) impact team adherence and injury risk in Canadian female youth football players: a cluster-randomised trial. British Journal of Sports Medicine, 47(8), 480-487. https://doi.org/10.1136/bjsports-2012-091887

Tarvainen, M. P., Niskanen, J. P., Lipponen, J. A., Ranta-Aho, P. O., \& Karjalainen, P. A. (2014). Kubios HRV-heart rate variability analysis software. Computer Methods and Programs in Biomedicine, 113(1), 210-220. https://doi.org/10.1016/j.cmpb.2013.07.024

\section{Corresponding author:}

\section{Prof. Yung-Sheng Chen}

Department of Exercise and Health Sciences, University of Taipei

No.101, Sec. 2, Jhongcheng Rd., Shihlin District, Taipei City 11153, Taiwan

Tel: +886 2 2871-8288 \#6405

Fax: +886 2 2875-1373

E-mail: yschen@utaipei.edu.tw 